\title{
Evaluation of a New Hierarchical Teaching Model for Pharmacy Students in Experiential Education
}

\author{
Laura Tsang, Minh-Hien Le, Vincent Teo, and Brian Hardy
}

\section{INTRODUCTION}

$\mathrm{P}$ harmacy students undergoing experiential education have traditionally been mentored by pharmacist preceptors on a one-to-one basis. However, major curriculum changes are placing greater emphasis on experiential education. For example, the new PharmD curriculum within the University of Toronto's Leslie Dan Faculty of Pharmacy requires Early Practice Experience (EPE) rotations of 160 h each after year 1 and year 2 (in place of the 12-h Early Hospital Experience [EHE] in year 2 of the previous program) and 36 weeks of experiential practice after year 3. Implementation of these changes has begun only within the past year (i.e., since mid-2012), and it will take some time to realize the full magnitude of the effect on staff workload at clinical sites; however, rotation requirements have already increased dramatically. In general, teaching sites strive to offer placements that are of high quality while accommodating the needs of pharmacist preceptors and clinical programs. This large increase in pharmacy student rotations has created a stress on the system and has forced sites to explore alternative methods of delivering experiential education.

The hierarchical model of teaching, with senior students mentoring junior students, is well known in the medical field. However, little is known about how effective or feasible this model might be for pharmacy students. Lindblad and others ${ }^{1}$ described peer-assisted learning for final-year pharmacy students on a 9-week rotation in a general medicine/stroke care unit. In that model, the first group of 3 students started their rotation at week 1 , with the second group of 3 students starting at week 5 . This allowed for overlap and mentoring by the more experienced students during weeks 5 to 9 . The second group then continued their rotation until week 13 . However, such peer-assisted learning is based on more experienced students facilitating the learning of a group of peers who are all at the same point in their education. It may not be suitable for models in which students are at different points in their academic programs.

Given the prospect of pharmacy students from different years completing rotations simultaneously, a hierarchical model may be one way to accommodate the increased demand for placements and to allow pharmacists to dedicate time to teaching in addition to their dispensing, clinical, and research duties. As described by Hall and others, ${ }^{2}$ pharmacy educators at Henry Ford Hospital in Detroit, Michigan, hypothesized that a hierarchical system similar to the medical model could be successful. Under this structure, pharmacy students at various levels (e.g., students, residents, fellows) would be under the supervision of an "attending" practitioner, who would be most responsible for the quality of patient care even as the students progressively assumed greater independence and more responsibilities. However, the changing availability of students from month to month was recognized as a potential barrier to full implementation of the system.

Here, we report an evaluation of a hierarchical teaching model in which fourth-year pharmacy students and residents mentored junior pharmacy students under the supervision of a pharmacist, in terms of perceived impact on learning, overall experience, and pharmacists' workload.

\section{METHODS}

Convenience sampling was used for this survey study. EHE students assigned to Sunnybrook Health Sciences Centre from January 2011 to May 2012 and their respective senior pharmacy student/resident mentors or pharmacist preceptors were invited to participate. All of the mentees were second-year students in the University of Toronto Leslie Dan Faculty of Pharmacy. Each 12-h EHE rotation was divided into two 6-h sessions or three 4-h sessions, as mutually agreed upon by the 
mentor and mentee. Because of the size of the class and the limited number of preceptors, students were assigned specific time windows to complete their rotations throughout the school year. Activities ranged from provision of direct patient care to learning about medication distribution. The mentors were fourth-year University of Toronto pharmacy students completing their 8-week Structured Practical Experience Program (SPEP) rotation or pharmacy residents or pharmacists at Sunnybrook Health Sciences Centre. Whenever possible, SPEP students or residents were assigned as mentors for the EHE rotations; when there were insufficient numbers of SPEP students or when residents were unavailable, EHE students were matched with a pharmacist preceptor. For this study, the term "mentoring" was defined as a relationship between 2 pharmacy trainees with the more experienced (senior) pharmacy student or resident teaching the less experienced (junior) pharmacy student. Sunnybrook Health Sciences Centre is a university-affiliated tertiary teaching hospital that provides acute care, long-term care, and outpatient services. In this study, all pharmacy students, residents, and pharmacists were part of the acute care pharmacy teams in cardiology, emergency medicine, and general medicine.

In all cases, the mentor or preceptor was responsible for organizing the entire hospital experience. A student assigned to a pharmacy resident or pharmacist spent $100 \%$ of the rotation with the mentor. A student assigned to an SPEP student spent virtually all of the rotation with the mentor, but also spent some time in group discussions with the attending pharmacist. In this case, the EHE student was the full responsibility of the SPEP student, who coordinated all dates, times, and activities. Hence, the traditional 1:1 pharmacist to student ratio shifted to a 1:1 "SPEP or resident mentor" to "EHE student" ratio, with some group learning time for EHE students. Figure 1 shows a schematic of the participants involved and the various relationships among mentees, mentors, and pharmacists.

Following each practice experience, surveys were distributed to mentees, mentors (SPEP students and residents), and pharmacists. The survey comprised 2 sections relating to the rotation. The first section was specific to whether the participant was a mentee, mentor, or pharmacist and used a combination of open-ended and Likert scale questions to assess quality, perceived impact on the SPEP student's or resident's learning, workload, and feasibility of the program. For pharmacists, data were also collected on dispensing and clinical time and number of previous students. In the second section, participants from all groups used a 5-point Likert scale to evaluate quality in relation to achievement of the specific goals of the EHE program. Descriptive statistics were used for baseline information and graphical results and to show differences between EHE students assigned to an SPEP or resident and those assigned to a pharmacist.

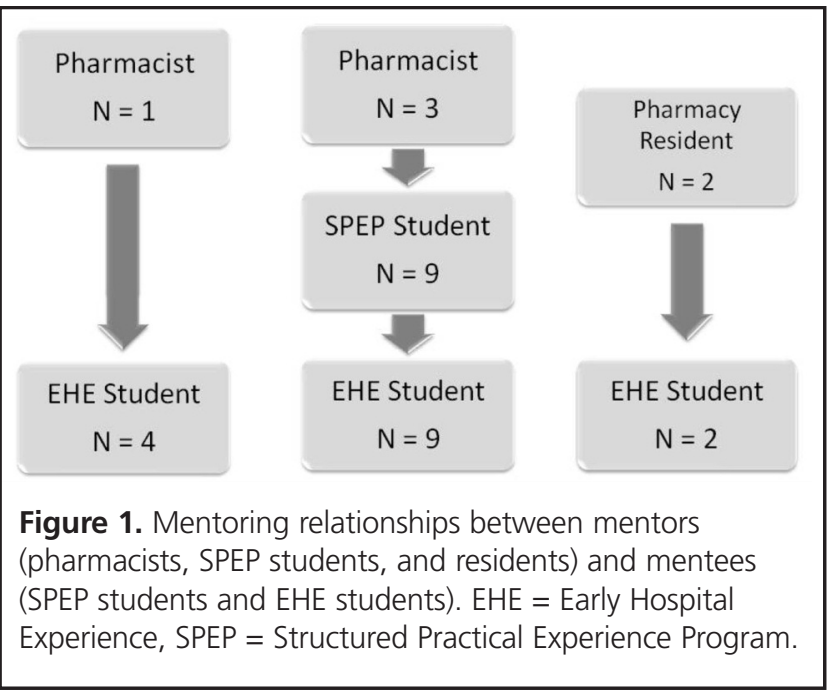

\section{RESULTS}

Pooled data were collected between January 2011 and May 2012. Overall, 15 EHE students, 9 SPEP students, 2 residents, and 3 pharmacists participated in the survey study. Of the 15 mentees, 9 (60\%) had SPEP mentors, $2(13 \%)$ had resident mentors, and $4(27 \%)$ were paired with a pharmacist. The mentees were actively involved in demonstrating devices and teaching patients about medications, collecting best possible medication histories, and researching drug information questions with the help of their respective mentors. All of the SPEP and resident mentors had previous pharmacy experience, almost all had been exposed to community practice, and more than half had been exposed to hospital practice. As well, 2 (18\%) of the 11 mentors had previous mentoring experience, including previous mentoring of an EHE student. The 3 pharmacists spent $60 \%$ of their time on clinical duties and $40 \%$ on dispensing responsibilities; they had on average 7.7 years of practice, including preceptor experience with students at various education levels.

\section{EHE Students with a Pharmacist Preceptor}

Through a series of open-ended questions, EHE students who had been paired with a pharmacist preceptor were asked whether they would have preferred an SPEP or resident mentor instead. Respondents described wanting an SPEP student as a mentor because SPEP students could provide a senior student perspective, would be easier to relate to, and would have greater availability. Respondents found the EHE rotation overwhelming at times and felt that an SPEP student could have provided support and acted as a bridge between second-year knowledge and hospital pharmacy practice. Respondents felt that a resident could have given insight into residency programs and that residents were more knowledgeable, in general, than SPEP students (Box 1). 


\section{Box 1. Selected EHE Student Responses to Open-Ended Survey Questions}

EHE students with a pharmacist preceptor

Do you think your experience would be enriched with a senior pharmacy student?

"Yes. SPEP students are usually eager to teach others, they are available consistently and are far more easy to relate to." "Yes, because you get to see the perspective from a senior student, in terms of position/profession. Also some education sessions may be overwhelming and hence to get an SPEP student's support to help understand the material would be good."

\section{EHE students with an SPEP or resident mentor}

Advantages of a student mentor

“Relatability ... can learn together ... knows pharmacy curriculum and therefore, can tailor learning experience to appropriate level ... provide suggestions/advice with respect to upper year pharmacy courses."

"Remembered school and therefore, has accurate assessment of our clinical knowledge."

"Student perspective was great, the mentor I had was very knowledgeable."

"Helpful, as was nice to help bridge our experience from school to practical experience."

"Makes EHE student feel comfortable in a new environment where they do not know everything ... gave student a chance to ask questions and seek advice about the pharmacy program, career options in pharmacy and other aspects of the health care industry."

Disadvantages of a student mentor

"Cannot answer every single question."

"May not have as much clinical knowledge in therapeutic area as a practising pharmacist."

"They may not be completely confident about all of the duties of a pharmacist in the hospital setting because it is a new experience for them as well."

EHE = Early Hospital Experience, SPEP = Structured Practical Experiential Program.

\section{EHE Students with an SPEP Student or Pharmacy Resident Mentor}

Students with an SPEP student or resident mentor were asked to rate a series of statements on a 5-point Likert scale. Among the 11 EHE students with a nonpharmacist mentor, 8 (73\%) strongly agreed and 3 (27\%) agreed that having an SPEP or resident mentor had enriched their rotation experience. As well, 7 (64\%) strongly agreed and 4 (36\%) agreed that the mentor had taught them things to which they would not normally have been exposed. With regard to whether they would have preferred a pharmacist-only mentorship (as opposed to a rotation with mentorship from a senior pharmacy student or resident), 2 (18\%) strongly disagreed, 3 (27\%) disagreed, 5 (45\%) were neutral, and only 1 (9\%) agreed.
Overall, 9 (82\%) strongly agreed and $2(18 \%)$ agreed that the hierarchical mentorship experience had been worthwhile; in addition, 7 (64\%) strongly agreed and 4 (36\%) agreed that they would recommend this model in the future.

Mentees were also asked to comment on the experience of having a student mentor through a series of open-ended questions (Box 1). Some advantages included the ability of EHE students to relate better to their mentors and the perception that these mentors better understood their mentees' knowledge level and the second-year curriculum. Disadvantages included SPEP mentors' lack of the therapeutic expertise and practical knowledge that would be expected of a pharmacist.

\section{SPEP Student and Pharmacy Resident Mentors}

SPEP students and residents were asked to assess the quality of the experience and their own workload using a 5-point Likert scale. Overall, 9 (82\%) of the 11 mentors strongly agreed and 2 (18\%) agreed that they found the experience worthwhile (Figure 2). In addition, 7 (64\%) strongly agreed and 4 (36\%) agreed that they would recommend this experience to future SPEP students and residents. In assessing their workload while working with a mentee, most of the SPEP and resident mentors disagreed (5 [45\%]) or strongly disagreed (1 [9\%]) that workload had increased significantly; 3 of the mentors $(27 \%)$ gave a neutral response, only $1(9 \%)$ agreed and $1(9 \%)$ strongly agreed that workload had increased significantly. In addition, respondents did not feel that working with EHE students impaired their own learning: 5 (45\%) strongly disagreed and 6 (55\%) disagreed that mentoring a student had significantly impaired their learning.

\section{Pharmacists}

Pharmacists strongly disagreed that learning by SPEP students and residents was disadvantaged with this new hierarchical model of experiential education. They also disagreed that their own workload was significantly increased or that the number of students on rotation resulted in space constraints on their units. In addition, all 3 pharmacists strongly agreed that they would be willing to use this model of training again in the future. In response to open-ended questions, the pharmacists noted the following as advantages of the program: allowing a greater number of EHE students to experience hospital practice and exposing EHE students to different areas of pharmacy practice. As well, the pharmacists felt that the program empowered the SPEP students and residents, assisting them in developing their own leadership, mentoring, and teaching skills. Reported disadvantages included an increase in workload for pharmacists when SPEP student mentors were absent and the possibility that student and resident mentors lacked sufficient experience and knowledge to work with the EHE students. 


\section{Attainment of EHE Learning Goals}

Mentees, mentors, and pharmacists were asked to assess whether the learning goals specified by the faculty's EHE program had been attained. For some goals, the assessments of achievement varied widely by respondent group (Table 1 ).

\section{DISCUSSION}

This study examined the quality and feasibility of a hierarchical teaching model in which fourth-year pharmacy students and residents mentored junior (second-year) pharmacy students under the supervision of a pharmacist. In assessing the quality of the EHE rotation in relation to program goals, EHE students and mentors alike indicated that the course outcomes could be met with an SPEP or resident mentor. A similar finding has been documented for medical models. In one study comparing fourth-year medical students with faculty members in the teaching of physical assessment to first-year students, the first-year students in the 2 groups achieved similar test scores, but those with fourth-year preceptors rated their experience

I gained teaching skills from this experience Having an EHE student significantly impaired my learning Have an EHE student significantly increased my workload

I would recommend this program in the future I felt confident in my mentoring skills I felt prepared and trained to mentor an EHE student I found this experience worthwhile

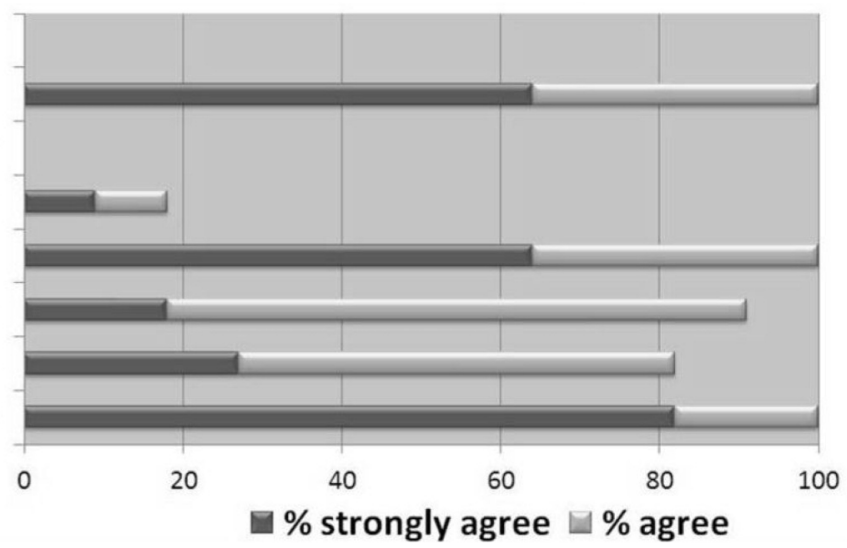

Figure 2. Results of survey of Structured Practical Experience Program students and pharmacy residents serving as mentors $(n=11)$. Data are shown only for ratings indicating agreement or strong agreement; neutral ratings and disagreement are not shown.

Table 1. Respondents' Assessment of Achievement of EHE Learning Goals

\section{EHE Learning Goal}

Mentee with
Pharmacist
Mentor
$(n=4)$

1. Observed and discussed how a medication order is processed from order decision to patient administration $4(100)$

\section{Mentee with SPEP or Resident Mentor} $(n=11)$
SPEP or Resident Mentor $(n=11)$
Pharmacist $(n=3)$

$3(27)$

$2(67)$

2. Observed and discussed drug distribution

$3(75)$

$3(75)$

$5(45)$

$5(45)$

$2(18)$

9 (82) $3(100)$ provides patient care (i.e., role of pharmacist, integration into health care team)

4. Observed or assisted in pharmacist activities, such as clarification of allergies, medication dose frequency

\begin{tabular}{|c|c|c|c|c|}
\hline 5. Viewed patient care documentation process & $2(50)$ & $7(64)$ & $6(55)$ & $2(67)$ \\
\hline 6. Discussed key components of medical chart & $2(50)$ & $7(64)$ & $8(73)$ & $2(67)$ \\
\hline 7. Discussed and observed medication reconciliation & $3(75)$ & $7(64)$ & $9(82)$ & $3(100)$ \\
\hline $\begin{array}{l}\text { 8. Observed and discussed key components of } \\
\text { medication discharge counselling }\end{array}$ & 0 & $2(18)$ & $7(64)$ & $2(67)$ \\
\hline $\begin{array}{l}\text { 9. Observed and discussed drug and patient } \\
\text { information resources available to a } \\
\text { hospital pharmacist }\end{array}$ & $2(50)$ & $5(45)$ & $6(55)$ & $1(33)$ \\
\hline 10. Attended pharmacist education session & $3(75)$ & $4(36)$ & $6(55)$ & 1 (33) \\
\hline
\end{tabular}

EHE $=$ Early Hospital Experience, SPEP $=$ Structured Practice Experience Program. 
higher than those with faculty preceptors. "Cognitive congruence" and "social congruence" have been postulated as likely explanations for this type of finding. ${ }^{3}$

In the current study, the mentees described their SPEP or resident mentors as being easier to relate to (relative to pharmacist preceptors), especially in the sometimesoverwhelming hospital environment. As well, they gained additional insights from their mentors that might not normally be covered by a pharmacist preceptor. Residents and SPEP mentors were able to connect real-world events with the inschool curriculum more proficiently than pharmacists, who might have graduated several years earlier and might not be as familiar with the current curriculum. Examples included relating clinical scenarios back to teachings from second-year laboratories, such as communication skills and device demonstrations. The SPEP students and residents were also able to link topics with knowledge that the junior students would gain in therapeutics courses in the upcoming (third) year of study. Many of the senior pharmacy students and residents shared their experiences in hospital practice in terms of career planning (e.g., initial thoughts, expectations of a hospital pharmacist, working culture). As these students were closer in age and education to the EHE students (than were the pharmacists), we surmised that such conversations might be more "frank" and that the EHE students might therefore relate better to their "near-peer" mentors. The concept of near-peer teaching involves a senior student teaching a junior student, with the senior student passing on knowledge and experiences while concurrently improving his or her own teaching skills. ${ }^{4}$ The unintended advantage of frank discussions between near-peer mentors could be especially valuable to junior students training under the new curriculum, whose experiential rotations will start earlier in their pharmacy education and who could benefit from the experiences of a near-peer teacher. It was also noted that mentors lacked the full extent of experience and knowledge of a practising pharmacist, but this situation was expected, given their level of training, and underlines the importance of involvement by an "attending pharmacist".

In assessing the quality of their experience, mentors described gaining valuable teaching skills and reported finding the process worthwhile. Residents had participated in a series of teaching skills workshops, which covered one-on-one preceptorship; however, SPEP student mentors had received no additional formal training related to mentorship. Nonetheless, all of the SPEP students felt well prepared to effectively mentor an EHE student and would recommend this program in the future. One possible reason for this sense of readiness might have been the 4- to 6-week, one-to-one preceptorship that SPEP students had undergone during their own rotations. A recent systematic review of peer-assisted teaching during medical school suggested that, in selected contexts, peer-assist- ed teaching models can achieve outcomes comparable to those achieved with faculty-based teaching. ${ }^{5}$ Therefore, it is postulated that the mentoring experience helped SPEP students to develop their own teaching abilities and confidence. As such, they may have gained pride in their accomplishments and may have been excited to share their new knowledge with junior pharmacy students.

With respect to workload, it was anticipated that the additional teaching component would affect mentors' learning and increase their workload, but survey responses showed that this was not the case. This unexpected finding might be explained by improved working efficiency after several weeks on rotation and the additional knowledge acquired in the process of teaching.

The pharmacist respondents reported that the hierarchical teaching model had minimal impacts on rotation quality for both mentees and mentors. Although pharmacists reported small increases in workload and space constraints in clinical areas, they also indicated that they would use this model of experiential training again. Pharmacists have traditionally been hesitant to accept additional EHE students on top of their current student, but these findings suggest that use of this hierarchical model could increase the capacity of teaching sites to accept students. As well, this study showed that a hierarchical teaching model could be successful for pharmacists with both dispensing and clinical responsibilities, especially given that dispensing duties may be a barrier to accepting more students.

It is expected that most Canadian pharmacy residents and postgraduate PharmD students will mentor some junior trainees. Some pharmacy residency and PharmD programs already incorporate teaching modules into their respective curricula. In a survey of US medical schools, 43 of 99 respondents indicated that they offered a formal "student-as-teachers" training program. ${ }^{6}$ To ensure continued success in hierarchical pharmacy teaching, formal clinical teaching programs will need to be incorporated earlier in the pharmacist training curriculum.

In the second part of the survey, participants were asked to assess whether the EHE program's learning goals had been achieved. For the majority of these goals, the respondent groups generally agreed in their assessments; however, for several goals, the assessments were inconsistent. For example, for the second learning goal, relating to drug distribution, $53 \%$ of all mentees (8/15) but only $14 \%$ (2 of 14 mentors and pharmacist preceptors) strongly agreed that the goal had been met (Table 1). At the study institution, mentors are assigned to clinical units and are shown the medication order process as part of orientation but are not formally trained in this process. This limited exposure might explain why mentors would be uncomfortable explaining this process and responding to questions from their mentees. Goals 3 and 8 involve discussion about how pharmacists provide patient care and discharge counselling. For both 
goals, a large percentage of pharmacists and SPEP and resident mentors strongly agreed that this goal was achieved; however, among the 15 mentees, only 8 (53\%) agreed that goal 3 had been achieved, and only 2 (13\%) agreed that goal 8 had been achieved. One possible explanation for this discrepancy may relate to the timing of EHE exposures in relation to clinical activities in the hospital: the majority of EHE exposures occurred in the afternoon (to allow for mentor learning in the mornings), but the majority of discharges and clinical rounds occur in the morning. As such, although the mentors may have explained the processes of patient care and discharge counselling, the mentees may not have had opportunities to observe these tasks being performed.

This study had several limitations. The sample size was relatively small (including only 3 pharmacists); as such, it was challenging to accurately assess workload and space constraints. As well, the majority of mentees had their EHE rotations after mentors had been in their rotations for 4 to 6 weeks. However, it may not always be possible to ensure that mentors have several weeks of experience in their own rotations before they begin mentoring more junior students, and it is difficult to determine how the experience would have differed if the mentees had arrived earlier in the mentors' rotation. Finally, the EHE exposures were only $12 \mathrm{~h}$ long, but under the new curriculum, in which rotations last for 1 month, the impact of hierarchical experiential rotations on mentor learning and workload may be different. One approach may be for several mentors to share a junior pharmacy student rotation to reduce the amount of time that each mentor is responsible for the student and thereby to reduce any impact on their own learning.

Future steps to expand on the results reported here could include gathering data for additional cohorts of mentors and mentees to ensure that the findings are reproducible and generalizable. This research could also be extended to examine the hierarchical relationship involving PharmD and year 1 and 2 EPE students under the supervision of an "attending pharmacist". As each of these rotations will be at least 4 weeks in duration, a study focused on rotation quality and feasibility would generate results addressing teaching scenarios that are closer to the real world. Finally, clinical efficiency, workload management, and patient satisfaction should also be further explored.

\section{CONCLUSIONS}

The new hierarchical teaching model described in this article was well received by EHE students, SPEP and resident mentors, and pharmacists. The EHE students preferred SPEP and resident mentors, as they were more accessible and were more familiar with the curriculum and career options. This study showed successful implementation of a hierarchical teaching model with supervision by pharmacists having both clinical and dispensing responsibilities. Ultimately, this approach will not only increase institutional capacity for student rotations, but will also maximize students' exposure to hospital practice.

\section{References}

1. Lindblad AJ, Howorko JM, Cashin RP, Ehlers CJ, Cox CE. Development and evaluation of a student pharmacist clinical teaching unit utilizing peer-assisted learning. Can J Hosp Pharm. 2011;64(6):446-50.

2. Hall K, Musing E, Miller DA, Tisdale JE. Experiential training for pharmacy students: time for a new approach. Can J Hosp Pharm. 2012;65(4):285-93.

3. Haist SA, Wilson JF, Brigham NL, Fosson SE, Blue AV. Comparing fourth-year medical students with faculty in the teaching of physical examination skills to first-year students. Acad Med. 1998;73(2):198-200.

4. Evans D, Cuffe T. Near-peer teaching in anatomy: an approach for deeper learning. Anat Sci Educ. 2009;2(5):227-33.

5. Yu TC, Wilson NC, Singh PP, Lemanu DP, Hawken SJ, Hill AG. Medical students-as-teachers: a systematic review of peer-assisted teaching during medical school. Adv Med Educ Pract. 2011;2:157-72.

6. Soriano RP, Blatt B, Coplit L, CichoskiKelly E, Kosowicz L, Newman L, et al. Teaching medical students how to teach: a national survey of students-as-teachers programs in U.S. medical schools. Acad Med. 2010;85(11):1725-31.

Laura Tsang, BSCPhm, ACPR, PharmD, is a pharmacist in the Schulich Heart Program, Sunnybrook Health Sciences Centre, Toronto, Ontario.

Minh-Hien Le, BScPhm, PharmD, is a pharmacist in General Internal Medicine at Sunnybrook Health Sciences Centre, Toronto, Ontario.

Vincent Teo, BSCPhm, ACPR, PharmD, is a pharmacist in the Emergency Department at Sunnybrook Health Sciences Centre, Toronto, Ontario.

Brian Hardy, PharmD, is Coordinator - Education and Clinical Programs, Department of Pharmacy, Sunnybrook Health Sciences Centre, and Associate Professor, Leslie Dan Faculty of Pharmacy, University of Toronto, Toronto, Ontario.

Competing interests: None declared.

Address correspondence to:

Laura Tsang

Department of Pharmacy

Sunnybrook Health Sciences Centre

Toronto ON M4N 3M5

e-mail: laura.tsang@sunnybrook.ca 